\title{
High-performance web services for querying gene and variant annotation
}

\author{
Jiwen Xin ${ }^{1 \dagger}$, Adam Mark ${ }^{1,2 \dagger}$, Cyrus Afrasiabi ${ }^{1 \dagger}$, Ginger Tsueng ${ }^{1}$, Moritz Juchler ${ }^{3}$, Nikhil Gopal ${ }^{3}$, Gregory S. Stupp ${ }^{1}$, \\ Timothy E. Putman ${ }^{1}$, Benjamin J. Ainscough ${ }^{4}$, Obi L. Griffith ${ }^{4}$, Ali Torkamani ${ }^{5,6}$, Patricia L. Whetzel ${ }^{7}$, \\ Christopher J. Mungall ${ }^{8}$, Sean D. Mooney ${ }^{3}$, Andrew I. Su ${ }^{1,6^{*}}$ and Chunlei $\mathrm{Wu}^{1 *}$
}

\begin{abstract}
Efficient tools for data management and integration are essential for many aspects of high-throughput biology. In particular, annotations of genes and human genetic variants are commonly used but highly fragmented across many resources. Here, we describe MyGene.info and MyVariant.info, high-performance web services for querying gene and variant annotation information. These web services are currently accessed more than three million times per month. They also demonstrate a generalizable cloud-based model for organizing and querying biological annotation information. MyGene.info and MyVariant.info are provided as high-performance web services, accessible at http://mygene.info and http://myvariant.info. Both are offered free of charge to the research community.
\end{abstract}

Keywords: Annotation, Gene, Variant, API, Cloud, Repository, Database

\section{Background}

The accumulation of biomedical knowledge is growing exponentially. There has been tremendous effort to structure research findings as annotations on biological entities (e.g., genes, genetic variants, and pathways). However, these annotations are fragmented among many resources that range greatly in terms of size, funding, and visibility (see, e.g., Ensembl [1], UniProt [2], PROSITE [3], and Reactome [4]). Tools for knowledge integration enable more efficient analysis of genome-scale data sets and discovery of relationships between biological entities.

Bioinformaticians facing data integration problems generally pursue one of two strategies: data warehousing or data federation. Data warehousing involves downloading flat files from various sources, writing parsers to process the files, and then loading the parsed data into a local database. This strategy has the advantage of very high performance, but it also requires significant initial effort to write the parsers and ongoing effort to keep the resource up to date. On the other hand, data federation works by accessing remote data resources through web

\footnotetext{
* Correspondence: asu@scripps.edu; cwu@scripps.edu

${ }^{\dagger}$ Equal contributors

${ }^{1}$ Department of Molecular and Experimental Medicine, The Scripps Research

Institute, 10550 North Torrey Pines Road, La Jolla, CA 92037, USA

Full list of author information is available at the end of the article
}

services. Federated data solutions are always up to date, but extra care is required to maintain the links, and large queries may take a long time to return due to server and network limitations. Moreover, the dependability of federated solutions is entirely dependent on the stability of the remote resources.

\section{Results and Discussion}

Here we present an alternative solution for integrating annotations on genes and human variants. MyGene.info and MyVariant.info are open source, high-performance, and continuously updated data application programming interfaces (APIs) for accessing comprehensive, structured gene and variant annotations. These resources are offered as cloud-based web service endpoints with the goal of providing "annotation as a service." MyGene.info and MyVariant.info are centralized repositories for aggregating and serving dispersed annotation data. Both are free of charge for use by the research community.

Other centralized resources for gene and variant annotations currently exist for genes (e.g., Bioconductor AnnotationData Packages [5] and Biomart [6]) and variants (e.g., ANNOVAR [7]). Relative to these existing tools, MyGene.info and MyVariant.info have several advantages. First, a local database is not required, reducing setup, administration, and maintenance costs. Second, 
we provide a high-performance API that allows realtime queries in analysis pipelines or web applications.

\section{Data integration}

MyGene.info is maintained as a comprehensive and upto-date repository for gene annotations. It integrates data from large, centralized databases as well as smaller, more specialized sources. Each data source has its own data importer, converting external data sources to a list of objects in JavaScript Object Notation (JSON) format. Each individual JSON object uses the National Center for Biotechnology (NCBI) gene ID [8] as the preferred primary key. The output of each parser is stored in a MongoDB instance with a timestamp recorded for each individual annotation object, and then all objects with the same primary key are combined into a single annotation object. In addition, we have built a scheduling system that automates the updates for each data source according to its own schedule (see Fig. 1). Currently, MyGene.info provides more than 200 gene-specific annotation fields ([9] and Additional file 1: Table S1) covering more than 13 million genes for more than 15,000 species [10].

MyVariant.info is built with a similar design and architecture as MyGene.info, but it focuses specifically on annotations of human genetic variants (Additional file 2: Figure S1). We utilize the nomenclature from the Human Genome Variation Society (HGVS) [11] to define the primary key in MyVariant.info (see Methods for specific rules). To prevent incorrect usage of HGVS IDs that could lead to potential errors in clinical interpretation, we also developed and implemented a variant validation function to ensure that all variant IDs included in MyVariant.info strictly follow HGVS guidelines. Currently, MyVariant.info contains more than 500 variant-specific annotation types ([12] and Additional file 3: Table S2) from dozens of resources, covering more than 334 million unique variants [13], including both coding and non-coding variants.

\section{Web services}

Performance, scalability, and stability are three key features of a successful web service provider. We built an Elasticsearch-based cluster to index the underlying JSON objects for both MyGene.info and MyVariant.info. This indexing engine provides both superior query performance and rich query syntax to handle a large amount of concurrent queries for a variety of use cases. The Elasticsearch cluster also comes with inherent scalability, so that we can dynamically adjust the size of the cluster to accommodate increased bandwidth as needed. For example, the MyGene.info system is currently hosted on the Amazon EC2 platform with four moderate servers, which based on our tests, can handle traffic from $>5000$ concurrent users for approximately 10,000 requests per minute. Greater than $95 \%$ of actual user requests take less than $30 \mathrm{~ms}$ to process (Additional file 2: Figure S2). This dynamic cluster setup also promotes stability of these services by allowing us to perform maintenance on individual nodes without bringing down the whole system.

Since the release of the v2 API (July 2013), MyGene.info has accumulated more than 133 million requests, and it

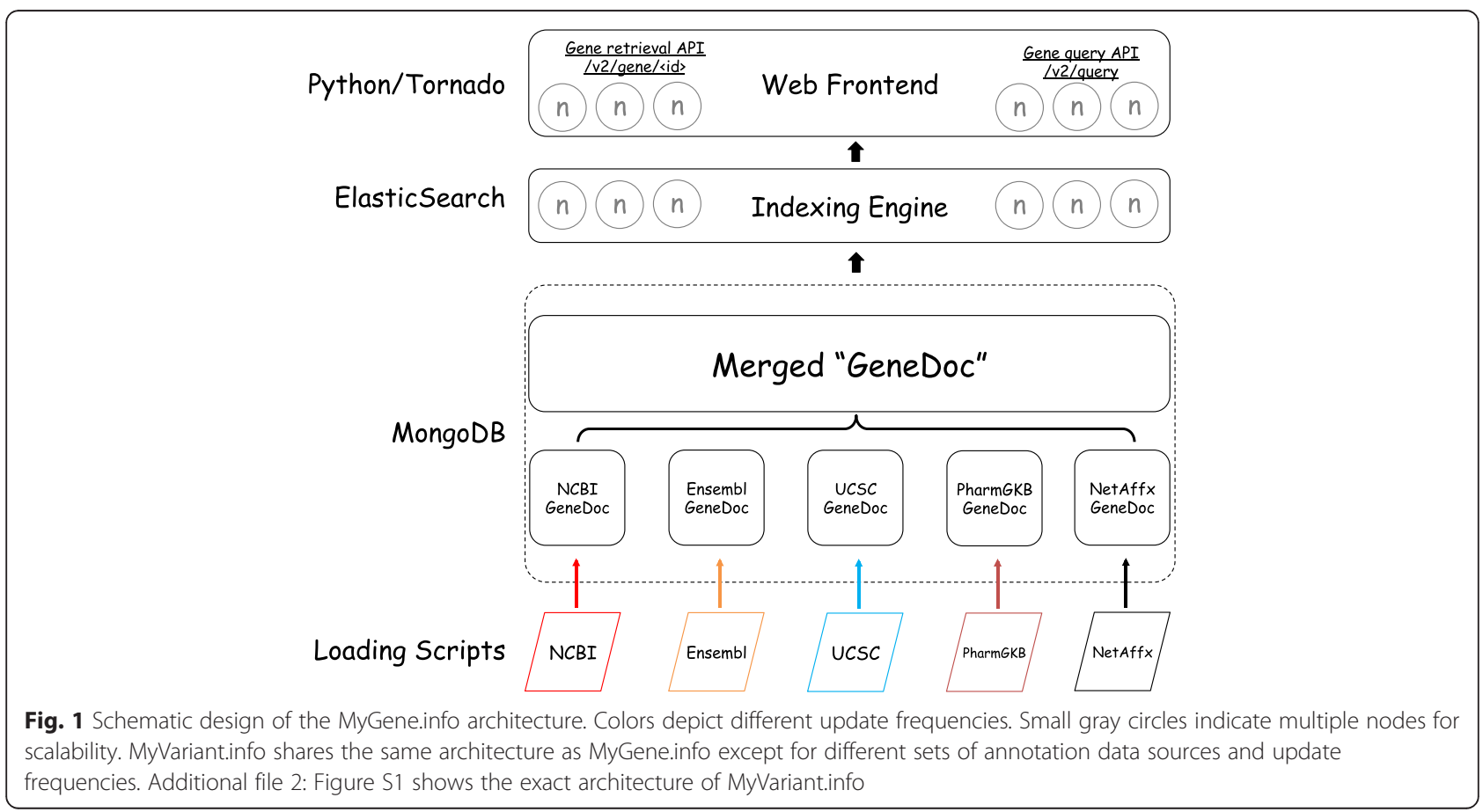


currently averages more than 3 million requests per month. Primary users include public resources like BioGPS [14], the Monarch Initiative [15], and CIViC [16]. In addition, numerous individual users incorporate MyGene.info into their bioinformatics analysis pipelines. According to our usage monitoring, approximately $30 \%$ of traffic comes from our BioGPS application, while $70 \%$ of traffic comes externally from more than 6000 unique IP addresses. The MyVariant.info API was launched in June 2015. To date, MyVariant.info has accumulated more than 1.5 million user queries.

\section{Use case}

To demonstrate their utility, we used MyVariant.info and MyGene.info to reimplement a typical analysis pipeline for interpreting exome sequencing results and identifying candidate genes for a rare Mendelian disease. In 2010, $\mathrm{Ng}$ et al. identified $D H O D H$ mutations as the genetic cause for Miller syndrome [17]. In their exome analysis, genes with nonsynonymous (NS) variants, splice acceptor and donor site mutations (SS), and coding indels (I) were first identified. Next, they filtered for genes containing NS/SS/I variants in all four sequenced samples. Previously observed variants in dbSNP129 [18], the 1000 Genomes Project [19], or HapMap were excluded. PolyPhen predictions [20] were used to prioritize variants that were predicted to be damaging. This process undoubtedly involved downloading, parsing, and analyzing annotation data from multiple databases, representing a significant investment of time and effort.

Using the MyGene.info and MyVariant.info R packages alone, we are able to implement an updated version of this pipeline: about 50 lines of code, requiring no local installation of variant annotation databases or software tools (see Fig. 2, [21], and Additional file 2: Supplementary Note 1). We first filtered for NS/SS/I variants and removed variants observed in the 1000 Genomes Project (as in [17]). We also incorporated an allele frequency filter based on data from the Exome Aggregation Consortium [22], filtered for candidate genes involved in metabolic processes ("GO:0008152") based on Gene Ontology annotations, and ranked candidate genes based on Combined Annotation Dependent Depletion (CADD) score [23] (an estimate of pathogenicity). After implementing this workflow for the Miller syndrome study, we were left with only five candidate genes, including the causal gene $D H O D H$. In addition, since MyVariant.info contains comprehensive and up-to-date variant annotations, it offers users the flexibility to further tailor this workflow based on other annotation fields (e.g., SIFT score [24], PolyPhen score [20], and clinical significance from ClinVar [25], etc.).
The utility of MyGene.info and MyVariant.info extends beyond this particular pipeline for exome sequencing analysis. Users can search for genes or variants using a wide variety of identifiers, and annotations can be retrieved for either single entities or lists. Users can also perform data-dependent queries (e.g., to find all variants with ExAC allele frequencies below 0.05 in the gene $B R C A 1)$. Queries can be performed through the webbased API or by using data access libraries for $\mathrm{R}$ or Python. These tools are flexible enough to incorporate into custom workflows, as well as responsive enough to perform real-time queries from other web applications. Finally, although MyGene.info and MyVariant.info focus on genes and human genetic variants, the underlying open source infrastructure is easily extensible to any type of biological entity.

\section{Implementation}

\section{Data sources}

Currently, MyGene.info contains data for about 200 annotation fields ([9], Additional file 1: Table S1) that were retrieved from eight public databases (Table 1), and MyVariant.info contains data for about 500 annotation fields ([12], Additional file 3: Table S2) that were retrieved from 14 public databases (Table 2). Our scheduler checks every data resource on a weekly basis, detects any changes to the source files, and applies incremental updates to our live servers. Annotation data from different databases exist in different formats, e.g., VCF, XML, and TSV. We wrote an individual data parser for all annotation data sources. Data parsers automatically import data from raw sources to facilitate regular updates where possible. All parser code for MyGene.info is available at Bitbucket [26]. All parser code for MyVariant.info is available at GitHub [27]. We also included sequence-based annotations generated from SnpEff [28] for each variant (if available). For example, multiple transcripts overlapping with a variant will be included as a list under the "snpeff.ann" field.

\section{Data integration}

The output of each data parser is a list of JSON objects. Each object contains an '_id' field as the primary key, which uniquely identifies a biological entity. MyGene.info uses the NCBI gene ID [8] as the preferred primary key, although the Ensembl gene ID [1] is used when no mapping to the NCBI gene ID is available.

For primary keys for variants, we used the nomenclature defined by the Human Genome Variation Society (HGVS) [11], as it is a recommended and widely accepted standard for describing variants. HGVS nomenclature allows multiple names to describe the same variant based on different reference sequences (e.g., genome assembly, transcript, or protein sequences). To define unique primary 


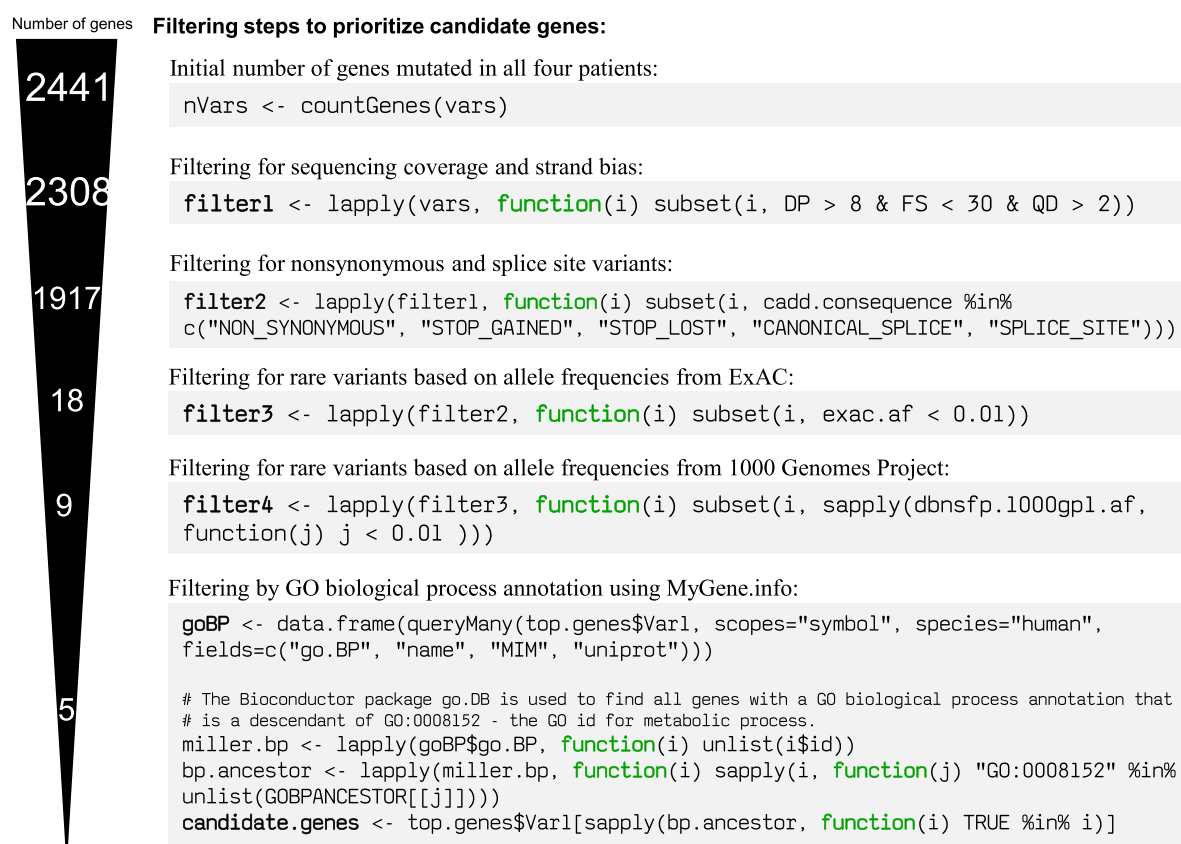

Fig. 2 The demo workflow for candidate gene prioritization using MyVariant.info and MyGene.info web services. We reimplemented five filtering steps in this workflow to prioritize candidate genes from a Miller syndrome study [17]. Selected R code is displayed for each filter step, using myvariant and mygene Bioconductor packages. The number of candidate genes left at each filtering step is displayed at the left side. The full code is available at https://github.com/sulab/myvariant.info/blob/master/docs/ipynb/myvariant_R_miller.ipynb, and also in Additional file 2: Supplementary Note 1

keys, we use the HGVS names based on the most commonly used reference genome assembly (currently hg19) and use $\operatorname{chr} 1, \operatorname{chr} 2, \ldots, \operatorname{chr} 22, \operatorname{chr} X, \operatorname{chr} Y$, and $\operatorname{chrMT}$ to represent chromosomes (e.g., chr11:g.111959693G>T - more examples are given in Additional file 2: Table S3). Although the primary keys of variant objects are based on genomic reference sequences, other valid HGVS names corresponding to alternate reference sequences (e.g., NC_000011.9:g.111959693G>T, NM_003002.2:c.274G>T) are also stored in each variant object and are indexed for queries.

We implemented a scheduling system to automate the updates for each data source. Currently, both
MyGene.info and MyVariant.info are updated weekly. The output of each parser is stored in a MongoDB instance with a timestamp recorded for each individual annotation object, and all objects with the same primary key ('_id' field) are combined into a single annotation object (see the examples in Additional file 2: Figure S3). This setup is advantageous, as it ensures the independent processing of each annotation source. Any single failure in the update process will not break the entire merging process, as in the case when a source file format changes and breaks a data parser. In that case, the last successful version of that failed source will be used until the parser is adapted to the changes.

Table 1 The list of data sources for MyGene.info. Column 1 lists the names of all eight data sources included in MyGene.info. Column 2 lists the version of each data source. Column 3 lists the URL for each data source

\begin{tabular}{llll}
\hline Source & Version & URL & Reference \\
\hline NCBI Entrez & $2015-10-24$ & http://www.ncbi.nlm.nih.gov/gquery/ & [40] \\
Ensembl & 82 & http://www.ensembl.org/ & [1] \\
UniProt & $2015-10-15$ & http://www.uniprot.org/ & [2] \\
NetAffx & na35 & https://www.affymetrix.com/analysis/index.affx & [41] \\
PharmGKB & $2015-10-05$ & https://www.pharmgkb.org/ & [42] \\
UCSC & $2015-10-20$ & https://genome.ucsc.edu/ & [43] \\
CPDB & 31 & http://cpdb.molgen.mpg.de/CPDB & [44] \\
RefSeq & 68 & http://www.ncbi.nlm.nih.gov/refseq/ & [45] \\
\hline
\end{tabular}


Table 2 The list of data sources for MyVariant.info. Column 1 lists the names of all 14 data sources included in MyVariant.info. Column 2 lists the version of each data source. Column 3 shows the number of variants from each data source included in MyVariant.info. Column 4 lists the URL for each data source

\begin{tabular}{|c|c|c|c|c|}
\hline Source & Version & No. of variants & URL & Reference \\
\hline dbNSFP & v3.0c & $82,030,830$ & https://sites.google.com/site/jpopgen/dbNSFP & [46] \\
\hline $\mathrm{dbSNP}$ & v144 & $145,132,257$ & http://www.ncbi.nlm.nih.gov/snp/ & [18] \\
\hline ClinVar & 2015-09 & 114,627 & http://www.ncbi.nlm.nih.gov/clinvar/ & [25] \\
\hline EVS & v2 & $1,977,300$ & http://evs.gs.washington.edu/EVS/ & [47] \\
\hline CADD & v1.2 & $163,690,986$ & http://cadd.gs.washington.edu/ & [23] \\
\hline MutDB & - & 420,221 & http://www.mutdb.org/ & [48] \\
\hline GWAS Catalog & From UCSC & 15,243 & http://www.ebi.ac.uk/gwas/ & [49] \\
\hline COSMIC & v68 from UCSC & $1,024,498$ & http://cancer.sanger.ac.uk/cosmic/ & {$[50]$} \\
\hline DOCM & - & 1119 & http://docm.genome.wustl.edu/ & [51] \\
\hline SNPedia & - & 5907 & http://www.snpedia.com/index.php/SNPedia & [52] \\
\hline EMVClass & - & 12,066 & http://geneticslab.emory.edu/emvclass/emvclass.php & [53] \\
\hline Wellderly & - & $21,240,519$ & http://www.stsiweb.org/wellderly/ & [54] \\
\hline EXAC & v0.3 & $10,195,872$ & http://exac.broadinstitute.org/ & [22] \\
\hline GRASP & V2.0.0.0 & $2,212,148$ & http://grasp.nhlbi.nih.gov/Overview.aspx & [55] \\
\hline
\end{tabular}

After the merging process, each JSON object contains all variant annotations aggregated from multiple sources. We then use Elasticsearch to index all fields within an annotation object so that users can make queries to retrieve annotations for their relevant genes or variants. Elasticsearch is a highly scalable, open source, full-text search and analytics engine. It provides a rich query syntax and inherent scalability to handle large-scale data queries in real time.

\section{Application programming interfaces}

On top of Elasticsearch, we built REST-based web services using the Tornado web framework. Tornado is a Python-based web framework built upon asynchronous networking technology that can provide tens of thousands of concurrent connections with a moderate server.

MyGene.info provides two simple-to-use REST-based web services: a gene query service and a gene annotation service. The gene query service allows users to query for gene annotations using any identifier or keyword, while the gene annotation service provides a convenient way to retrieve gene-centric annotations when gene IDs (NCBI gene IDs or Ensembl gene IDs) are available.

MyVariant.info also provides two REST-based web services: a variant query service that returns matching variant objects based on user queries and a variant retrieval service that returns the matching variant object(s) for a given ID (HGVS names, RS IDs, etc.).

Batch mode is supported by both services for querying a large list of IDs or query terms in one request. Both query services provide rich query syntax suitable for a variety of use cases. For example, users can query for matching variant annotation objects by various criteria, such as genomic ranges, prediction score cutoffs, exact field matching, and keyword search in a text field. Users also have the option of specifying the subset of fields they want to return if they do not require the entire annotation object. More complicated queries can be constructed by combining multiple query terms with Boolean operators or by conducting faceting, with aggregations for special use cases. Information on the types of queries that are enabled by MyGene.info can be found at [29]. Information on the types of queries that are enabled by MyVariant.info can be found at [30].

\section{Use case demonstration}

We reanalyzed the exome sequencing data generated by $\mathrm{Ng}$ et al. for their Miller syndrome study [17]. Genomic DNA from four patients were sequenced in this study. FASTQ files were processed according to the GATK best practice [29]. Individual samples were aligned to the hg19 reference genome using BWA 0.7.10. Variants were called using GATK 3.3 HaplotypeCaller, and quality scores were recalibrated using GATK VariantRecalibrator. The Bioconductor packages myvariant and mygene were utilized to demonstrate a streamlined application for variant filtering and prioritization of candidate genes in rare Mendelian disorders.

\section{Query interface}

Although the initial design of MyGene.info and MyVariant.info APIs is targeted to bioinformatics 
developers, we also consider it a necessity to provide a query interface for our APIs, which can serve both as the demo interface for developers and the entry points for general researchers. We have implemented the initial versions of our easy-to-use query interface for both APIs: http://mygene.info/demo and http:// myvariant.info/demo. They can be easily accessed from each site's landing page.

\section{Python and $\mathrm{R}$ clients}

A Python client and an $\mathrm{R}$ client are available for both MyGene.info and MyVariant.info. The Python client for MyGene.info can be downloaded at the Python Package Index [30].

The $\mathrm{R}$ client for MyGene.info is released as part of Bioconductor [31]. The Python client for MyVariant.info can be downloaded at the Python Package Index [32], and the $\mathrm{R}$ client for MyVariant.info is also released as part of Bioconductor [33].

\section{Availability and requirements}

MyGene.info web services can be accessed at http:// mygene.info, with the interactive API documentation at http://mygene.info/v2/api/ and the full documentation at http://docs.mygene.info.

MyVariant.info web services can be accessed at http:// myvariant.info, with the interactive API documentation at http://myvariant.info/v1/api/ and the full documentation at http://docs.myvariant.info.

MyGene.info and MyVariant.info are both open source projects (licensed under the Apache License, Version 2.0). The source code for these projects can be found at [34] (MyGene.info web frontend), [35] (MyGene.info data backend), and [36] (MyVariant.info). They have also been deposited to Zenodo (https://zenodo.org/) with assigned DOIs: 10.5281/zenodo.48146 (MyGene.info web frontend) [37], 10.5281/zenodo.48145 (MyGene.info data backend) [38], and 10.5281/zenodo.48086 (MyVariant.info) [39].

Exome sequence data from two siblings with Miller syndrome and two unrelated affected individuals were provided by $\mathrm{Ng}$ et al. [17] through the database of Genotypes and Phenotypes (dbGaP) under accession number [dbGaP:phs000244.v1.p1].

\section{Conclusions}

MyGene.info (http://mygene.info) and MyVariant.info (http://myvariant.info) are provided as high-performance web services for querying gene and variant annotation information, currently with over three million user requests per month.

\section{Additional files}

Additional file 1: Table S1. The gene-specific annotation fields available from MyGene.info. (XLS $32 \mathrm{~kb}$ )

Additional file 2: Includes Figure S1: Schematic design of MyVariant.info; Figure S2: Histograms of the request time for MyGene.info; Figure S3: JSON annotation object examples; Table S3: Examples of HGVS nomenclature; Supplementary Note 1: IPython notebook for Miller syndrome study. (PDF $946 \mathrm{~kb}$ )

Additional file 3: Table S2. The variant-specific annotation fields available from MyVariant.info. (XLS 58 kb)

Ethics approval

Ethics approval was not needed for this study.

\section{Competing interests}

The authors declare that they have no competing interests.

\section{Authors' contributions}

AIS and CW conceived the project. JX, AM, CA, and CW implemented the core software. GT coordinated the project outreach. MJ, NG, GSS, TEP, BJA, OLG, AT, PLW, CJM, and SDM contributed either code or data to the project. JX, AM, AIS, and CW wrote the manuscript with input from all authors. AIS, SDM, and CW directed this project. All authors read and approved the final manuscript.

\section{Acknowledgements}

The authors would like to acknowledge the valuable input from Dr. Robin Haw at the First Network of BioThings Hackathon.

\section{Funding}

This work was supported by the US National Institute of Health (grants U01HG008473 to CW, GM083924 and U54GM114833 to AIS, U01HG006476 to AT, and K22CA188163 to OLG). This work was also supported by the Scripps Translational Science Institute with an NIH-NCATS Clinical and Translational Science Award (CTSA; 5 UL1 TR001114).

\section{Author details}

${ }^{1}$ Department of Molecular and Experimental Medicine, The Scripps Research Institute, 10550 North Torrey Pines Road, La Jolla, CA 92037, USA. ${ }^{2}$ Current address: Avera Cancer Institute, 11099 North Torrey Pines Road, La Jolla, CA 92037, USA. ${ }^{3}$ Department of Biomedical Informatics and Medical Education, The University of Washington, Box SLU-BIME 358047, Seattle, WA 98195, USA. ${ }^{4}$ McDonnell Genome Institute, Washington University School of Medicine, 4444 Forest Park Ave, St. Louis, MO 63108, USA. ${ }^{5}$ Department of Integrative Structural and Computational Biology, The Scripps Research Institute, 10550 North Torrey Pines Road, La Jolla, CA 92037, USA. ${ }^{6}$ The Scripps Translational Science Institute, The Scripps Research Institute, 10550 North Torrey Pines Road, La Jolla, CA 92037, USA. ${ }^{7}$ Center for Research in Biological Systems, University of California San Diego, 9500 Gilman Drive, La Jolla, CA 92093, USA. ${ }^{8}$ Lawrence Berkeley National Laboratory, 1 Cyclotron Road, Berkeley, CA 94720, USA.

Received: 7 January 2016 Accepted: 14 April 2016

Published online: 06 May 2016

References

1. Flicek P, Amode MR, Barrell D, Beal K, Billis K, Brent S, et al. Ensembl 2014. Nucleic Acids Res. 2014;42:D749-755.

2. UniProt C. Activities at the Universal Protein Resource (UniProt). Nucleic Acids Res. 2014:42:D191-198.

3. Sigrist CJ, de Castro E, Cerutti L, Cuche BA, Hulo N, Bridge A, et al. New and continuing developments at PROSITE. Nucleic Acids Res. 2013:41:D344-347.

4. Croft D, Mundo AF, Haw R, Milacic M, Weiser J, Wu G, et al. The Reactome pathway knowledgebase. Nucleic Acids Res. 2014;42:D472-477.

5. Gentleman RC, Carey VJ, Bates DM, Bolstad B, Dettling M, Dudoit S, et al. Bioconductor: open software development for computational biology and bioinformatics. Genome Biol. 2004;5:R80. 
6. Smedley D, Haider S, Durinck S, Pandini L, Provero P, Allen J, et al. The BioMart community portal: an innovative alternative to large, centralized data repositories. Nucleic Acids Res. 2015;43:W589-598.

7. Wang K, Li M, Hakonarson H. ANNOVAR: functional annotation of genetic variants from high-throughput sequencing data. Nucleic Acids Res. 2010;38: e164.

8. Brown GR, Hem V, Katz KS, Ovetsky M, Wallin C, Ermolaeva O, et al. Gene: a gene-centered information resource at NCBI. Nucleic Acids Res. 2015;43: D36-42.

9. MyGene.info annotation fields. 2013.http://docs.mygene.info/en/latest/doc/ data.htm|\#available-fields. Accessed 25 Mar 2016.

10. MyGene.info metadata information. http://mygene.info/metadata. Accessed 25 Mar 2016.

11. den Dunnen JT, Antonarakis SE. Mutation nomenclature extensions and suggestions to describe complex mutations: a discussion. Hum Mutat. 2000;15:7-12.

12. MyVariant.info annotation fields. 2015. http://docs.myvariant.info/en/latest/ doc/data.html\#available-fields. Accessed 25 Mar 2016

13. MyVariant.info metadata information. http://myvariant.info/metadata. Accessed 25 Mar 2016

14. Wu C, Macleod I, Su Al. BioGPS and MyGene.info: organizing online, gene-centric information. Nucleic Acids Res. 2013;41:D561-565.

15. Mungall CJ, Washington NL, Nguyen-Xuan J, Condit C, Smedley D, Kohler S, et al. Use of model organism and disease databases to support matchmaking for human disease gene discovery. Hum Mutat. 2015;36:979-84. Accessed 25 Mar 2016.

16. Clinical Intepretations of Variants in Cancer. https://civic.genome.wustl.edu/.

17. Ng SB, Buckingham KJ, Lee C, Bigham AW, Tabor HK, Dent KM, et al. Exome sequencing identifies the cause of a mendelian disorder. Nat Genet. 2010;42:30-5.

18. Sherry ST, Ward MH, Kholodov M, Baker J, Phan L, Smigielski EM, et al. dbSNP: the NCBI database of genetic variation. Nucleic Acids Res. 2001;29:308-11.

19. Genomes Project Consortium, Abecasis GR, Auton A, Brooks LD, DePristo MA, Durbin RM, et al. An integrated map of genetic variation from 1,092 human genomes. Nature. 2012;491:56-65.

20. Adzhubei IA, Schmidt S, Peshkin L, Ramensky VE, Gerasimova A, Bork P, et al. A method and server for predicting damaging missense mutations. Nat Methods. 2010;7:248-9

21. A demo use case of MyVariant.info and MyGene.info services in IPython Notebook. https:/github.com/sulab/myvariant.info/blob/master/docs/ipynb/ myvariant_R miller.jpynb. Accessed 25 Mar 2016

22. Exome Aggregation Consortium, Lek M, Karczewski K, Minikel E, Samocha K, Banks E, Fennell T, O'Donnell-Luria A, Ware J, Hill A, et al. Analysis of protein-coding genetic variation in 60,706 humans. bioRxiv. 2015 http://dx.doi.org/10.1101/030338

23. Kircher M, Witten DM, Jain P, O'Roak BJ, Cooper GM, Shendure J. A general framework for estimating the relative pathogenicity of human genetic variants. Nat Genet. 2014;46:310-5.

24. Kumar P, Henikoff S, Ng PC. Predicting the effects of coding non-synonymous variants on protein function using the SIFT algorithm. Nat Protoc. 2009;4:1073-81.

25. Landrum MJ, Lee JM, Riley GR, Jang W, Rubinstein WS, Church DM, et al. ClinVar: public archive of relationships among sequence variation and human phenotype. Nucleic Acids Res. 2014;42:D980-985.

26. MyGene.info parser code. https://bitbucket.org/sulab/mygene.hub/src/ default/src/dataload/sources/. Accessed 25 Mar 2016

27. MyVariant.info parser code. https://github.com/sulab/myvariant.info/tree/ master/src/dataload/contrib/. Accessed 25 Mar 2016

28. Cingolani P, Platts $A$, le Wang $L$, Coon $M$, Nguyen $T$, Wang $L$, et al. A program for annotating and predicting the effects of single nucleotide polymorphisms, SnpEff: SNPs in the genome of Drosophila melanogaster strain w1118; iso-2; iso-3. Fly (Austin). 2012;6:80-92.

29. MyGene.info Query Types. http://docs.mygene.info/en/latest/doc/query_ service.html. Accessed 25 Mar 2016.

30. MyVariant.info Query Types. http://docs.myvariant.info/en/latest/doc/ variant_query_service.html. Accessed 25 Mar 2016.

31. Mark A, Thompson R, Wu C. MyGene.info R client. 2014. http:// bioconductor.org/packages/release/bioc/html/mygene.html. Accessed 25 Mar 2016.

32. MyVariant.info Python client. https://pypi.python.org/pypi/myvariant/.
33. Mark A. MyVariant.info R client. 2015.http://bioconductor.org/packages/ release/bioc/html/myvariant.html.

34. MyGene.info web frontend source code. https://bitbucket.org/sulab/ mygene.info.

35. MyGene.info data backend source code. https://bitbucket.org/sulab/ mygene.hub.

36. MyVariant.info source code. https://github.com/sulab/myvariant.info.

37. Xin J, et al. MyGene.info web frontend component. Zenodo. 2016. http://dx. doi.org/10.5281/zenodo.48146. Accessed 25 Mar 2016.

38. Xin J, et al. MyGene.info data backend component. Zenodo. 2016. http://dx doi.org/10.5281/zenodo.48145.

39. Xin J, et al. MyVariant.info - build fb2a871. Zenodo. 2016. http://dx.doi.org/ 10.5281/zenodo.48086.

40. Maglott D, Ostell J, Pruitt KD, Tatusova T. Entrez Gene: gene-centered information at NCBI. Nucleic Acids Res. 2005:33:D54-58.

41. Liu G et al. NetAffx: Affymetrix probesets and annotations. Nucleic Acids Res. 2003:31:82-6.

42. Whirl-Carrillo M, McDonagh EM, Hebert JM, Gong L, Sangkuhl K, Thorn CF, et al. Pharmacogenomics knowledge for personalized medicine. Clin Pharmacol Ther. 2012:92:414-7.

43. Kent WJ, Sugnet CW, Furey TS, Roskin KM, Pringle TH, Zahler AM, et al. The human genome browser at UCSC. Genome Res. 2002;12:996-1006.

44. Kamburov A, Stelzl U, Lehrach H, Herwig R. The ConsensusPathDB interaction database: 2013 update. Nucleic Acids Res. 2013:41:D793-800.

45. Pruitt KD, Brown GR, Hiatt SM, Thibaud-Nissen F, Astashyn A, Ermolaeva O, et al. RefSeq: an update on mammalian reference sequences. Nucleic Acids Res. 2014:42:D756-763.

46. Liu X, Jian X, Boerwinkle E. dbNSFP v2.0: a database of human nonsynonymous SNVS and their functional predictions and annotations. Hum Mutat. 2013;34:E2393-2402.

47. Exome Variant Server, NHLBI GO Exome Sequencing Project (ESP), Seattle, WA. http://evs.gs.washington.edu/EVS/. Accessed 25 Mar 2016.

48. Singh A, Olowoyeye A, Baenziger PH, Dantzer J, Kann MG, Radivojac P, et al MutDB: update on development of tools for the biochemical analysis of genetic variation. Nucleic Acids Res. 2008;36:D815-819.

49. Welter D, MacArthur J, Morales J, Burdett T, Hall P, Junkins $H$, et al. The NHGRI GWAS Catalog, a curated resource of SNP-trait associations. Nucleic Acids Res. 2014;42:D1001-1006.

50. Forbes SA, Beare D, Gunasekaran P, Leung K, Bindal N, Boutselakis H, et al. COSMIC: exploring the world's knowledge of somatic mutations in human cancer. Nucleic Acids Res. 2015;43:D805-811.

51. Database of Curated Mutations. http://docm.genome.wustl.edu. Accessed 25 Mar 2016.

52. Cariaso M, Lennon G. SNPedia: a wiki supporting personal genome annotation interpretation and analysis. Nucleic Acids Res. 2012;40:D1308-1312.

53. Bean $L$, Tinker SW, da Silva C, Hegde MR. Free the data: one laboratory's approach to knowledge-based genomic variant classification and preparation for EMR integration of genomic data. Hum Mutat. 2013;34:1183-8

54. STSI Variant Browser - Wellderly. http://www.stsiweb.org/wellderly. Accessed 25 Mar 2016.

55. Leslie R, O'Donnell CJ, Johnson AD. GRASP: analysis of genotype-phenotype results from 1390 genome-wide association studies and corresponding open access database. Bioinformatics. 2014;30:1185-194.

\section{Submit your next manuscript to BioMed Central and we will help you at every step:}

- We accept pre-submission inquiries

- Our selector tool helps you to find the most relevant journal

- We provide round the clock customer support

- Convenient online submission

- Thorough peer review

- Inclusion in PubMed and all major indexing services

- Maximum visibility for your research

Submit your manuscript at www.biomedcentral.com/submit 